\title{
MARX-STROHHÄCKER DIFFERENTIAL SUBORDINATION SYSTEMS
}

\author{
SANFORD S. MILLER AND PETRU T. MOCANU
}

\begin{abstract}
Let $f(z)=z+a_{2} z^{2}+\cdots$ be analytic in the unit disc $U$ and let $k(z)=z /(1-z)$. The classic Marx-Strohhäcker result, that a convex (univalent) function $f$ is starlike of order $\frac{1}{2}$, can be written in terms of differential subordinations as

$$
z f^{\prime \prime}(z) / f^{\prime}(z) \prec z k^{\prime \prime}(z) / k^{\prime}(z) \Rightarrow z f^{\prime}(z) / f(z) \prec z k^{\prime}(z) / k(z) .
$$

The authors determine general conditions on $k$ for which this relation holds. They also determine a different set of general conditions on $k$ for which

$$
z f^{\prime}(z) / f(z) \prec z k^{\prime}(z) / k(z) \Rightarrow f(z) / z \prec k(z) / z .
$$

Finally, differential subordinations with starlike superordinate functions are considered.
\end{abstract}

1. Introduction. Let $f$ be analytic in the unit disc $U=\{z:|z|<1\}$. The function $f$, with $f^{\prime}(0) \neq 0$, is convex (univalent) if and only if $\operatorname{Re}\left[z f^{\prime \prime}(z) / f^{\prime}(z)+1\right]>$ 0 in $U$. The function $f$, with $f(0)=0$ and $f^{\prime}(0) \neq 0$, is starlike (univalent) if and only if $\operatorname{Re}\left[z f^{\prime}(z) / f(z)\right]>0$ in $U$. If, in addition, $\operatorname{Re}\left[z f^{\prime}(z) / f(z)\right]>\frac{1}{2}$ then $f$ is said to be starlike of order $\frac{1}{2}$ [1, Vol. 1, p. 137].

The classic result of Marx [3] and Strohhäcker [10] asserts that a convex function is starlike of order $\frac{1}{2}$, that is

$$
\operatorname{Re}\left[z f^{\prime \prime}(z) / f^{\prime}(z)+1\right]>0, z \in U \Rightarrow \operatorname{Re}\left[z f^{\prime}(z) / f(z)\right]>\frac{1}{2}, z \in U .
$$

We can simplify this relation by expressing it in terms of subordination. If $F$ and $G$ are analytic in $U$, then $F$ is subordinate to $G$, written $F \prec G$ or $F(z) \prec G(z)$, if $G$ is univalent, $F(0)=G(0)$ and $F(U) \subset G(U)$.

If we let $k(z)=z /(1-z)$, then (1) can be rewritten as

$$
z f^{\prime \prime}(z) / f^{\prime}(z) \prec z k^{\prime \prime}(z) / k^{\prime}(z) \Rightarrow z f^{\prime}(z) / f(z) \prec z k^{\prime}(z) / k(z) .
$$

This Marx-Strohhäcker differential subordination system of first type holds for other functions $k$; in [5, p. 194] and in [7, Corollary 2.3] it is shown that (2) also holds when $k(z)=z /(1-z)^{2}$ is the Koebe function. In $\S 2$ of this article we determine general conditions on $k$ for which (2) holds.

If $k$ is the Koebe function, then Marx [3] and Strohhäcker [10] also showed that

$$
z f^{\prime}(z) / f(z) \prec z k^{\prime}(z) / k(z) \Rightarrow f(z) / z \prec k(z) / z .
$$

Received by the editors December 30, 1985.

1980 Mathematics Subject Classification (1985 Revision). Primary 30C80; Secondary 30C45, 34A20, 34A40.

Key words and phrases. Differential subordination, superordinate, convex function, starlike function, univalent function.

The first author acknowledges support received from the International Research Exchange Board through its exchange program with the Romanian National Council of Science and Technology. 
$\S 3$ deals with general first order differential subordinations of the form $\psi\left(p(z), z p^{\prime}(z)\right)$ $\prec h(z)$, where $h$ is a starlike function. In addition, as a special application of these results we determine conditions on $k$ for which the Marx-Strohhäcker differential subodination system of second type, as given in (3), holds.

We close this section with two lemmas that will be used in subsequent sections.

LEMMA 1. Let $F$ be analytic in $U$ and let $G$ be analytic and univalent on $\bar{U}$, with $F(0)=G(0)$. If $F$ is not subordinate to $G$, then there exist points $z_{0} \in U$ and $\varsigma_{0} \in \partial U$, and an $m \geq 1$ for which $F\left(|z|<\left|z_{0}\right|\right) \subset G(U)$,

(i) $F\left(z_{0}\right)=G\left(s_{0}\right)$, and

(ii) $z_{0} F^{\prime}\left(z_{0}\right)=m_{\zeta_{0}} G^{\prime}\left(\varsigma_{0}\right)$.

The proof of a more general form of this lemma may be found in [4, Lemma 1].

The second lemma deals with the concept of a subordination chain. A function $L(z, t), z \in U, t \geq 0$, is a subordination chain if $L(\cdot, t)$ is analytic and univalent in $U$ for all $t \geq 0, L(z, \cdot)$ is continuously differentiable on $[0, \infty)$ for all $z \in U$, and $L\left(z, t_{1}\right) \prec L\left(z, t_{2}\right)$ when $0 \leq t_{1} \leq t_{2}$.

LEMMA $2[8, \mathrm{8} .159]$. The function $L(z, t)=a_{1}(t) z+\cdots$, with $a_{1}(t) \neq 0$ for all $t \geq 0$, is a subordination chain if and only if $\operatorname{Re}[z(\partial L / \partial z) /(\partial L / \partial t)]>0$ for $z \in U$ and $t \geq 0$.

\section{Marx-Strohhäcker differential subordination systems of first type.}

THEOREM 1. Let $q$ be univalent in $U$, with $q(0)=1$. Set $Q(z)=z q^{\prime}(z) / q(z)$, $h(z)=q(z)+Q(z)$ and suppose that

(i) $Q$ is starlike in $U(\log q$ is convex in $U)$, and

(ii) $\operatorname{Re}\left[z h^{\prime}(z) / Q(z)\right]=\operatorname{Re}\left[q(z)+z Q^{\prime}(z) / Q(z)\right]>0, z \in U$.

If $B$ is an analytic function on $U$ such that

$$
B(z) \prec q(z)+z q^{\prime}(z) / q(z)=h(z),
$$

then the analytic solution $p$ of the differential equation

$$
z p^{\prime}(z)+B(z) p(z)=1 \quad(p(0)=1)
$$

satisfies $p \prec 1 / q$.

ProOF. Conditions (i) and (ii) imply that the function $h$ is univalent (closeto-convex) [1, Vol. 2, p. 2], $h(0)=1$, and hence (4) is well defined with $B(0)=1$. If we let $b(z)=\int_{0}^{z}[B(t)-1] t^{-1} d t$, then (5) has an analytic solution given by $p(z)=\int_{0}^{z} e^{b(t)} d t / z e^{b(z)}$. Condition (i) implies that $q(z) \neq 0$ in $U$. Hence the function $1 / q$ is analytic and univalent in $U$.

Without loss of generality we can assume $q$ is univalent and $q(z) \neq 0$ on $\bar{U}$. If not, then we can replace $q, B$, and $p$ by $q_{r}(z)=q(r z), B_{r}(z)=B(r z)$, and $p_{r}(z)=p(r z)$ respectively, where $0<r<1$. These new functions satisfy the conditions of the theorem on $\bar{U}$. We would then prove $p_{r} \prec 1 / q_{r}$, and by letting $r \rightarrow 1^{-}$we obtain $p \prec 1 / q$.

The function

$$
L(z, t)=h(z)+t Q(z)=q(z)+(1+t) z q^{\prime}(z) / q(z)
$$


is analytic in $U$ for all $t \geq 0$, and it is continuously differentiable on $[0, \infty)$ for all $z \in U$. Since $Q^{\prime}(0)=q^{\prime}(0) \neq 0$ we have $\partial L / \partial z(0, t)=q^{\prime}(0)(2+t) \neq 0$ for $t \geq 0$. From (ii) we obtain

$$
\operatorname{Re}\left[z \frac{\partial L}{\partial z} / \frac{\partial L}{\partial t}\right]=\operatorname{Re}\left[q(z)+(1+t) \frac{z Q^{\prime}(z)}{Q(z)}\right] \geq \operatorname{Re} \frac{z Q^{\prime}(z)}{Q(z)}>0
$$

for $z \in U$ and $t \geq 0$. Hence by Lemma 2, $L(z, t)$ is a subordination chain and so we have $L\left(z, t_{1}\right) \prec L\left(z, t_{2}\right)$ for $0 \leq t_{1} \leq t_{2}$. From (6) we have $L(z, 0)=h(z)$ and so we obtain

$$
L(\zeta, t) \notin h(U)
$$

for $|\zeta|=1$ and $t \geq 0$.

Now assume that $p \nprec 1 / q$. From Lemma 1 there exist points $z_{0} \in U$ and $\varsigma_{0} \in \partial U$, and an $m \geq 1$ such that $p\left(z_{0}\right)=1 / q\left(\varsigma_{0}\right)$ and $z_{0} p^{\prime}\left(z_{0}\right)=-m \varsigma_{0} q^{\prime}\left(\varsigma_{0}\right) / q^{2}\left(\varsigma_{0}\right)$. Using these results together with (5) and (6) we obtain

$$
B\left(z_{0}\right)=\frac{1}{p\left(z_{0}\right)}-\frac{z_{0} p^{\prime}\left(z_{0}\right)}{p\left(z_{0}\right)}=q\left(\varsigma_{0}\right)+\frac{m \varsigma_{0} q^{\prime}\left(\varsigma_{0}\right)}{q\left(\varsigma_{0}\right)}=L\left(\varsigma_{0}, m-1\right) .
$$

By using (7) we deduce that $B\left(z_{0}\right) \notin h(U)$, which contradicts (4). Hence $p \prec 1 / q$, completing the proof of the theorem.

We now turn our attention to considering the Marx-Strohhäcker differential subordination system of first type as given in (2). If we let $p(z)=z f^{\prime}(z) / f(z)$ and $q(z)=z k^{\prime}(z) / k(z)$ then $(2)$ becomes

$$
p(z)+z p^{\prime}(z) / p(z) \prec q(z)+z q^{\prime}(z) / q(z) \Rightarrow p(z) \prec q(z) .
$$

This differential subordination result was considered in [5, Theorem 3]. Before using this general result to prove (2), however, we have to first prove that $z f^{\prime}(z) / f(z)$ is analytic in $U$, a condition which does not follow directly from the left side of (2).

THEOREM 2. Let $q$ satisfy the conditions of Theorem 1 and let

$$
k(z)=z \exp \int_{0}^{z}[q(t)-1] t^{-1} d t .
$$

If $f(z)=z+a_{2} z^{2}+\cdots$ is analytic in $U$, and

$$
z f^{\prime \prime}(z) / f^{\prime}(z) \prec z k^{\prime \prime}(z) / k^{\prime}(z)
$$

then $z f^{\prime}(z) / f(z)$ is analytic in $U$ and $z f^{\prime}(z) / f(z) \prec z k^{\prime}(z) / k(z)$.

PROOF. From (8) we obtain

$$
q(z)=\frac{z k^{\prime}(z)}{k(z)} \quad \text { and } \quad h(z)=q(z)+\frac{z q^{\prime}(z)}{q(z)}=1+\frac{z k^{\prime \prime}(z)}{k^{\prime}(z)} .
$$

Thus $z k^{\prime}(z) / k(z)$ is univalent, and by (ii) of Theorem 1 we see that $z k^{\prime \prime}(z) / k^{\prime}(z)$ is also univalent. Since condition (9) implies $f^{\prime}(z) \neq 0$, the function $B(z)=$ $1+z f^{\prime \prime}(z) / f^{\prime}(z)$ is analytic in $U$, and satisfies $B \prec h$. For this particular $B$ equation (5) has the analytic solution $p(z)=f(z) / z f^{\prime}(z)$. Since all the conditions of Theorem 1 are satisfied, we have $p \prec 1 / q$. Since $1 / q \neq 0$ this implies that $p \neq 0$, and so $1 / p(z)=z f^{\prime}(z) / f(z)$ is analytic in $U$. In addition, from $p \prec 1 / q$ and $1 / q \neq 0$ we obtain $1 / p \prec q$, which yields the desired conclusion $z f^{\prime}(z) / f(z) \prec z k^{\prime}(z) / k(z)$. 
EXAMPLES. (a) The function $q(z)=[1+(1-2 \alpha) z] /(1-z)$, with $0 \leq \alpha<1$, satisfies (i) and (ii) of Theorem 1 . From Theorem 2 we obtain $k(z)=k(\alpha, z)=$ $z /(1-z)^{2(1-\alpha)}$ and

$$
\begin{aligned}
\frac{z f^{\prime \prime}(z)}{f^{\prime}(z)} & \prec \frac{z k^{\prime \prime}(\alpha, z)}{k^{\prime}(\alpha, z)}=\frac{2 z(1-\alpha)(2+(1-2 \alpha) z)}{(1-z)(1+(1-2 \alpha) z)} \\
& \Rightarrow \frac{z f^{\prime}(z)}{f(z)} \prec \frac{z k^{\prime}(\alpha, z)}{k(\alpha, z)}=\frac{1+(1-2 \alpha) z}{1-z} .
\end{aligned}
$$

The cases $\alpha=\frac{1}{2}$ and $\alpha=0$ correspond to the functions $z /(1-z)$ and $z /(1-z)^{2}$ respectively, which were mentioned in $\S 1$.

(b) The function $q(z)=z /\left(1-e^{-z}\right)$, which is a convex (univalent) function $[6$, p. 70] satisfies $q(0)=1$ and

$$
Q(z)=z q^{\prime}(z) / q(z)=1+z /\left(1-e^{z}\right)=1-q(-z) .
$$

Since $q$ is convex we conclude that $Q$ is also convex. By using (1) we obtain $\operatorname{Re} z Q^{\prime}(z) / Q(z)>\frac{1}{2}$, which implies condition (i) of Theorem 1. Condition (ii) also holds since

$$
\operatorname{Re}\left[q(z)+z Q^{\prime}(z) / Q(z)\right] \geq 1 /(e-1)+1 / 2>0 .
$$

Hence by Theorem 2 we obtain $k(z)=e^{z}-1$ and

$$
\begin{aligned}
& z f^{\prime \prime}(z) / f^{\prime}(z) \prec z k^{\prime \prime}(z) / k^{\prime}(z)=z \\
& \quad \Rightarrow z f^{\prime}(z) / f(z) \prec z k^{\prime}(z) / k(z)=z /\left(1-e^{-z}\right) .
\end{aligned}
$$

(c) The function $q(z)=[(1+z) /(1-z)]^{\beta}$, with $0<\beta \leq 1$, satisfies (i) and (ii) of Theorem 1 and by Theorem 2 we have

$$
\frac{z f^{\prime \prime}(z)}{f^{\prime}(z)} \prec\left[\frac{1+z}{1-z}\right]^{\beta}+\frac{z^{2}+2 \beta z-1}{1-z^{2}} \Rightarrow \frac{z f^{\prime}(z)}{f(z)} \prec\left[\frac{1+z}{1-z}\right]^{\beta} .
$$

(d) Employing the function $q(z)=e^{\lambda z}$ in Theorem 1 we obtain $Q(z)=\lambda z$ and $q(z)+z Q^{\prime}(z) / Q(z)=e^{\lambda z}+1$. Condition (i) is satisfied and condition (ii) will be satisfied if $\operatorname{Re}\left[e^{\lambda z}+1\right]>0$. A simple calculation shows that this condition holds for $|\lambda| \leq r_{0}=2.183 \ldots$, where $r_{0}=y_{0} / \mathrm{SIN} y_{0}$ and $y_{0}$ is the smallest root of $\operatorname{COS} y+\exp (y \operatorname{COT} y)=0$. Hence by Theorem 2, for $|\lambda| \leq r_{0}=2.183 \ldots$, we obtain $k(z)=z \exp \int_{0}^{z}\left(e^{\lambda t}-1\right) t^{-1} d t$ and

$$
\begin{gathered}
z f^{\prime \prime}(z) / f^{\prime}(z) \prec z k^{\prime \prime}(z) / k^{\prime}(z)=e^{\lambda z}+\lambda z-1 \\
\Rightarrow z f^{\prime}(z) / f(z) \prec z k^{\prime}(z) / k(z)=e^{\lambda z} .
\end{gathered}
$$

(e) The function $q(z)=1+\lambda z,|\lambda| \leq 1$, satisfies the conditions of Theorems 1 and 2 and we obtain $k(z)=z e^{\lambda z}$ and

$$
\frac{z f^{\prime \prime}(z)}{f^{\prime}(z)} \prec \frac{z k^{\prime \prime}(z)}{k^{\prime}(z)}=\frac{\lambda z(2+\lambda z)}{(1+\lambda z)} \Rightarrow \frac{z f^{\prime}(z)}{f(z)} \prec \frac{z k^{\prime}(z)}{k(z)}=1+\lambda z .
$$

In the special case $\lambda=1$ this simplifies to

$$
z f^{\prime \prime}(z) / f^{\prime}(z) \prec z(2+z) /(1+z) \Rightarrow\left|z f^{\prime}(z) / f(z)-1\right|<1 .
$$

This is an improvement of the result $\left|f^{\prime \prime}(z) / f^{\prime}(z)\right| \leq 3 / 2 \Rightarrow\left|z f^{\prime}(z) / f(z)-1\right|<1$ proved in $\left[\mathbf{7}\right.$, Theorem 3] and the result $\left|z f^{\prime \prime}(z) / f^{\prime}(z)+1\right|<2 \Rightarrow\left|z f^{\prime}(z) / f(z)-1\right|<$ 1 proved in [5, Theorem 5]. 


\section{Differential subordinations with starlike superordinate function.}

THEOREM 3. Let $H(z)=z+a_{2} z^{2}+\cdots$ be starlike in $U$ and let $\psi: C^{2} \rightarrow C$ be a function of the form $\psi(r, s)=w(r) \cdot s^{\alpha}$ with $\alpha>0$. In addition, suppose that

$$
\psi\left(q(z), z q^{\prime}(z)\right)=w(q(z)) \cdot\left(z q^{\prime}(z)\right)^{\alpha}=H(z)
$$

has a univalent solution $q$. If $p$ is analytic in $U$, with $p(0)=q(0)$, then

$$
w(p(z)) \cdot\left(z p^{\prime}(z)\right)^{\alpha} \prec H(z) \Rightarrow p(z) \prec q(z) .
$$

PROOF. If $p \nprec q$ then by Lemma 1 there exist points $z_{0} \in U$ and $\varsigma_{0} \in \partial U$, and an $m \geq 1$ such that $p\left(z_{0}\right)=q\left(\varsigma_{0}\right)$ and $z_{0} p^{\prime}\left(z_{0}\right)=m_{\varsigma_{0}} q^{\prime}\left(\varsigma_{0}\right)$. Using these results in (10) we obtain

$$
w\left(p\left(z_{0}\right)\right) \cdot\left(z_{0} p^{\prime}\left(z_{0}\right)\right)^{\alpha}=w\left(q\left(\varsigma_{0}\right)\right) \cdot\left(m \varsigma_{0} q^{\prime}\left(\varsigma_{0}\right)\right)^{\alpha}=m^{\alpha} H\left(\varsigma_{0}\right) .
$$

Since $H(U)$ is starlike and $m^{\alpha} \geq 1$ we obtain $w\left(p\left(z_{0}\right)\right) \cdot\left(z_{0} p^{\prime}\left(z_{0}\right)\right)^{\alpha} \notin H(U)$. This contradicts (10) and so we must have $p \prec q$.

Note that $(10)$ requires $\psi(q(0), 0)=0$. The special case $\psi(r, s)=r^{1-\alpha} s^{\alpha}$, with $0<\alpha \leq 1$, satisfies Theorem 3, with $p(0)=q(0)$, and was considered in [4, p. 169]. For the purposes of this paper, Theorem 3 is most interesting when $\psi(r, s)=s / r$. Applying the theorem in this case we obtain

THEOREM 4. Let $H(z)=z+a_{2} z^{2}+\cdots$ be starlike in $U$ and suppose that

$$
Q(z)=\exp \int_{0}^{z} H(t) t^{-1} d t
$$

is univalent. If $p$ is analytic in $U$, with $p(0)=1$, then

$$
z p^{\prime}(z) / p(z) \prec z Q^{\prime}(z) / Q(z)=H(z) \Rightarrow p(z) \prec Q(z) .
$$

COROLlaRY 4.1. If $H$ is a convex (univalent) and $\operatorname{Re} H(z)>-1$, then $Q$ as given in (12) is univalent and (13) holds.

ProOF. From (1) and (12) we obtain

$$
\operatorname{Re}\left[1+z Q^{\prime \prime}(z) / Q^{\prime}(z)\right]=\operatorname{Re}\left[H(z)+z H^{\prime}(z) / H(z)\right]>-1+\frac{1}{2}=-\frac{1}{2},
$$

which shows that $Q$ is univalent [9, Corollary 3].

If we set $H(z)=q(z)-1$ and $p(z)=f(z) / z$ we obtain

COROLlaRY 4.2. Let $q$ be convex with $\operatorname{Re} q(z)>0$ and let

$$
k(z)=z \exp \int_{0}^{z}[q(t)-1] t^{-1} d t .
$$

If $f(z)=z+a_{2} z^{2}+\cdots$ is analytic in $U$, then

$$
z f^{\prime}(z) / f(z) \prec z k^{\prime}(z) / k(z) \Rightarrow f(z) / z \prec k(z) / z .
$$

REMARKS. 1. This relation is precisely the one that the Koebe function satisfied in (3) of $\S 1$. The corollary lists sufficient conditions on $k$ to ensure that the MarxStrohhäcker differential subordination system of second type holds. 
2. The functions $q$ used in Examples (a), (b), (c), and (e) also satisfy the conditions of the corollary, whereas $q$ in (d) does not for $|\lambda|>1$. As a result, for Example (a) we have

$$
\frac{z f^{\prime \prime}(z)}{f^{\prime}(z)} \prec \frac{z k^{\prime \prime}(\alpha, z)}{k^{\prime}(\alpha, z)} \Rightarrow \frac{z f^{\prime}(z)}{f(z)} \prec \frac{z k^{\prime}(\alpha, z)}{k^{\prime}(\alpha, z)} \Rightarrow \frac{f(z)}{z} \prec \frac{k(\alpha, z)}{z},
$$

where $k(\alpha, z)=z /(1-z)^{2(1-\alpha)}$ and $0 \leq \alpha<1$.

For Example (b) we have

$$
z f^{\prime \prime}(z) / f^{\prime}(z) \prec z \Rightarrow z f^{\prime}(z) / f(z)<z /\left(1-e^{-z}\right) \Rightarrow f(z) / z<\left(e^{z}-1\right) / z
$$

and for Example (c) we have the extension

$$
\frac{z f^{\prime}(z)}{f(z)} \prec\left[\frac{1+z}{1-z}\right]^{\beta} \Rightarrow \frac{f(z)}{z} \prec \exp \int_{0}^{z}\left(\left[\frac{1+t}{1-t}\right]^{\beta}-1\right) t^{-1} d t
$$

for $0<\beta \leq 1$. In the special case $\beta=\frac{1}{2}$ this simplifies to

$$
\frac{z f^{\prime}(z)}{f(z)} \prec\left[\frac{1+z}{1-z}\right]^{1 / 2} \Rightarrow \frac{f(z)}{z} \prec \frac{2 \exp \left(\mathrm{SIN}^{-1} z\right)}{1+\left(1-z^{2}\right)^{1 / 2}}=Q(z) .
$$

From the last corollary we know that $\operatorname{Re}\left(1+z Q^{\prime \prime}(z) / Q^{\prime}(z)\right)>-\frac{1}{2}$ and that $Q$ is univalent. To show that the function $Q$ is also convex it is sufficient to show $\operatorname{Re}\left(1+z Q^{\prime \prime}(z) / Q^{\prime}(z)\right) \geq 0$ for $|z|=1$. Since

$$
1+z Q^{\prime \prime}(z) / Q^{\prime}(z)=[(1+z) /(1-z)]^{1 / 2}-1+\frac{1}{2}\left[(1-z)^{-1}+\left(1-z^{2}\right)^{-1 / 2}\right],
$$

if we let $z=e^{i t}, 0 \leq t \leq \pi$, we obtain

$$
(1+z) /(1-z)=i \operatorname{COT}(t / 2), \quad 1-z^{2}=-2 i e^{i t} \operatorname{SIN} t
$$

and

$\operatorname{Re}\left[1+z Q^{\prime \prime}(z) / Q^{\prime}(z)\right]=\left(\frac{1}{2} \operatorname{COT}(t / 2)\right)^{1 / 2}-\frac{3}{4}+[\operatorname{COS}(t / 2)+\operatorname{SIN}(t / 2)] / 4(\operatorname{SIN} t)^{1 / 2}$.

If we let $x=(\operatorname{COT}(t / 2))^{1 / 2} \geq 0$, we obtain

$$
\operatorname{Re}\left[1+z Q^{\prime \prime}(z) / Q^{\prime}(z)\right]=\left(5 x^{2}-3 \sqrt{2} x+1\right) / 4 \sqrt{2} x>0 .
$$

We close this section by considering another differential subordination having a starlike superordinate function. In [4, p. 170] and in [2, p. 192] it is shown that if $h$ is convex and $p$ is analytic in $U$, then

$$
p(z)+z p^{\prime}(z) \prec h(z) \Rightarrow p(z) \prec z^{-1} \int_{0}^{z} h(t) d t .
$$

We can replace the superordinate function $h$ with a function of the form $[(1+z) /(1-z)]^{\alpha}$. For $\alpha>1$ this function is not convex, but is starlike with respect to 1 .

THEOREM 5. Let $\beta_{0}=1.21872 \ldots$ be the solution of $\beta \pi=3 \pi / 2-\mathrm{TAN}^{-1} \beta$ and let $\alpha=\alpha(\beta)=\beta+2 \mathrm{TAN}^{-1} \beta / \pi$ for $0<\beta \leq \beta_{0}$. If $p$ is analytic in $U$, with $p(0)=1$, then

$$
p(z)+z p^{\prime}(z) \prec\left[\frac{1+z}{1-z}\right]^{\alpha} \Rightarrow p(z) \prec\left[\frac{1+z}{1-z}\right]^{\beta}
$$


PROOF. Note that $\beta \pi \leq 3 \pi / 2-\mathrm{TAN}^{-1} \beta$ and $\beta \leq 3-\alpha$ for $0<\beta \leq \beta_{0}$. If we let $h(z)=[(1+z) /(1-z)]^{\alpha}$ and $q(z)=[(1+z) /(1-z)]^{\beta}$ then $h(U)$ and $q(U)$ are domains given by the sectors $|\arg h|<\alpha \pi / 2$ and $|\arg g|<\beta \pi / 2$ respectively. We need to show that $p \prec q$. If this is not true, then there exist points $z_{0} \in U$ and $\varsigma_{0} \in \partial U$ such that $p\left(z_{0}\right)=q\left(\varsigma_{0}\right)$ and $p\left(|z|<\left|z_{0}\right|\right) \subset q(U)$. We need to consider separately the case $p\left(z_{0}\right) \neq 0$ which corresponds to a point on one of the rays on the sector $q(U)$, and the case $p\left(z_{0}\right)=0$ which corresponds to the corner of the sector.

Case (i). If $p\left(z_{0}\right) \neq 0$ then $\varsigma_{0} \neq \pm 1$. If we let $r i=\left(1+\varsigma_{0}\right) /\left(1-\varsigma_{0}\right)$ and use Lemma 1 we obtain

$$
\begin{aligned}
p\left(z_{0}\right)+z_{0} p^{\prime}\left(z_{0}\right) & =q\left(\zeta_{0}\right)+m \zeta_{0} q^{\prime}\left(\zeta_{0}\right)=(r i)^{\beta}-(r i)^{\beta-1} m \beta\left(1+r^{2}\right) / 2 \\
& =(r i)^{\beta}\left[1+i m \beta\left(1+r^{2}\right) / 2 r\right],
\end{aligned}
$$

and

$$
\arg \left(p\left(z_{0}\right)+z_{0} p^{\prime}\left(z_{0}\right)\right)=\beta \pi / 2+\mathrm{TAN}^{-1}(m \beta(r+1 / r) / 2) .
$$

Since TAN $^{-1}$ is an increasing function and $m \geq 1$, by considering the cases $r>0$ and $r<0$ we obtain

$$
\beta \pi / 2+\mathrm{TAN}^{-1} \beta \leq\left|\arg \left(p\left(z_{0}\right)+z_{0} p^{\prime}\left(z_{0}\right)\right)\right| \leq \beta \pi / 2+\pi / 2 .
$$

Using the fact that $\beta \pi \leq 3 \pi / 2-$ TAN $^{-1} \beta$ for $0<\beta \leq \beta_{0}$ and the definition of $\alpha$ we obtain

$$
\alpha \pi / 2 \leq\left|\arg \left(p\left(z_{0}\right)+z_{0} p^{\prime}\left(z_{0}\right)\right)\right| \leq 2 \pi-\alpha \pi / 2 .
$$

This implies that $p\left(z_{0}\right)+z_{0} p^{\prime}\left(z_{0}\right)$ lies outside the sector $h(U)$, and since this contradicts the hypothesis of the theorem we must have $p \prec q$.

Case (ii). The case $p\left(z_{0}\right)=0$ can occur only if $\beta \geq 1$, since $\beta<1$ implies that the sector angle of $q(U)$ is less than $\pi$ and $p\left(|z|=\left|z_{0}\right|\right)$ cannot pass through such a corner without itself having a corner. If $p^{\prime}\left(z_{0}\right) \neq 0$, then the smallest possible value of $\arg \left(z_{0} p^{\prime}\left(z_{0}\right)\right)$ is given by $(2 \pi-\beta \pi / 2)-\pi / 2$, which occurs when $p\left(|z|=\left|z_{0}\right|\right)$ is tangent to the lower ray of the sector $q(U)$. The largest possible value of $\arg \left(z_{0} p^{\prime}\left(z_{0}\right)\right)$ is given by $\beta \pi / 2+\pi / 2$, which occurs when $p\left(|z|=\left|z_{0}\right|\right)$ is tangent to the upper ray of the sector $q(U)$. Using these limitations and the fact that $\beta \leq 3-\alpha$ we obtain

$$
\alpha \pi / 2 \leq \arg \left(p\left(z_{0}\right)+z p^{\prime}\left(z_{0}\right)\right) \leq 2 \pi-\alpha \pi / 2,
$$

which gives the same contradiction as Case (i). The subcase $p^{\prime}\left(z_{0}\right)=0$ also yields a contradiction since $p\left(z_{0}\right)+z_{0} p^{\prime}\left(z_{0}\right)=0 \notin h(U)$.

REMARKS. If we take $\beta=1$ in Theorem 5 we obtain $\alpha(1)=\frac{3}{2}$ and

$$
p(z)+z p^{\prime}(z) \prec\left[\frac{1+z}{1-z}\right]^{3 / 2} \Rightarrow p(z) \prec \frac{1+z}{1-z} .
$$

In this case the angle of the sector $h(U)$ is $270^{\circ}$. The case with the largest angle corresponds to $\alpha_{0}=\alpha\left(\beta_{0}\right)=\alpha(1.218 \ldots)=1.781 \ldots$ In this case the angle of the sector $h(U)$ is $320.62 \ldots{ }^{\circ}$, while the angle of sector $q(U)$ is $219.36 \ldots \circ$.

\section{REFERENCES}

1. A. W. Goodman, Univalent functions. Vols. 1, 2, Mariner, Tampa, Florida, 1983.

2. D. J. Hallenbeck and S. Ruscheweyh, Subordination by convex functions, Proc. Amer. Math. Soc. 52 (1975), 191-195. 
3. A. Marx, Unteruchungen über schlicht Abbildungen, Math. Ann. 107 (1932/33), 40-67.

4. S. Miller and P. Mocanu, Differential subordinations and univalent functions, Michigan Math. J. 28 (1981), 151-171.

5. _ On some classes of first-order differential subordinations, Michigan Math. J. 32 (1985), 185-195.

6. P. Mocanu, Convexity of some particular functions, Studia Univ. Babeş-Bolyai Math. 29 (1984), 70-73.

7. 31 (1986), 231-235.

8. Ch. Pommerenke, Univalent functions, Vanderhoeck \& Ruprecht, Göttingen, 1975.

9. K. Sakaguchi, A note on p-valent functions, J. Math. Soc. Japan 14 (1962), 312-321. MR 26 \#6383.

10. E. Strohhäcker, Beiträqe zur Theorie der schlicten Funktionen, Math. Z. 37 (1933), 356380.

Department of Mathematics and Computer Science, State University of NEW YORK, BROCKPORT, NEW YORK 14420

FACUlty of MAthematics, BABEŞ-Bolyai University, 3400 Cluj-Napoca, RomaNiA (Current address of P. T. Mocanu)

Current address (S. S. Miller): Department of Mathematics, University of California, Berkeley, California 94720 